\title{
In situ measurement of CBR concrete modulus. Choice of the type of dam in morocco (static loads and earthquake cases).
}

\author{
TlemçaniM.K. ${ }^{1}$, Ait Brahim L. ${ }^{2}$, TardieuB.3 \\ ${ }^{1,3}$ Université Mohammed V, Faculté des \\ sciences, Unité de recherche GEORISK, LGRN, 4 Avenue Ibn Battouta \\ Rabat-Agdal, BP 1014, Rabat, Maroc. \\ ${ }^{2}$ Expert international-France
}

\begin{abstract}
The Young's modulus of roller compacted concrete is rarely measured. However, it has a direct influence on the dam behavior and especially for static loads during earthquakes. In many cases, static and dynamic calculations are done with modules copied from other study. This dam was studied using in situ module measurements that contribute to a choice of the type of dam and the choice of materials from borrow pits near the dam.
\end{abstract}

Dans le cas d'un site de barrage au Maroc, il a été nécessaire de mesurer le module d'Young de façon à améliorer la qualité de la prévision des modèles statiques et dynamiques utilisés pour prévoir le comportement du barrage.

La médiocrité des caractéristiques de la fondation a conduit ce barrage ainsi que d'autres barrages récents au Maroc, au choix du type du barrage, il s'agit d'un barrage poids rectiligne de profil quasi symétrique. Un profil quasi symétrique permet d'améliorer l'angle d'incidence des forces appliquées par la gravité et par l'eau et de diminuer l'état de contrainte dans la fondation.

Les calculs montrent que le niveau de contrainte est faible dans la fondation, elle est également faible dans le corps du barrage. Ce qui justifie le choix d'un béton faiblement dosé en ciment et en matériaux fins et dont les agrégats proviennent de zones d'emprunt situées à proximité immédiate du barrage, malgré des caractéristiques mécaniques moyennes dues à un contenu schisteux notable.

Pour évaluer le module du BCR, différents types de mesures ont été effectués durant la construction du barrage : des mesures directes sur éprouvettes réalisées au laboratoire, des essais sur carottes extraites du corps du barrage, des essais cross-hole réalisés dans le corps du barrage. Ces différents essais et les résultats obtenus sont exposés après une brève description du projet.

\section{DESCRIPTION DU PROJET}

Il s'agit d'un barrage poids en BCR au profil à parement amont avec un fruit de $0.5 \mathrm{H} / 1 \mathrm{~V}$ et à parement aval avec un fruit de $0.7 \mathrm{H} / 1 \mathrm{~V}$. La hauteur maximale sur fond de fouilles est d'environ $98.20 \mathrm{~m}$ et la longueur en crête d'est de $383.30 \mathrm{~m}$. Le volume du barrage est d'environ de $1000000 \mathrm{~m} 3$ de béton.

La coupe type originale du barrage est définie sur la base des caractéristiques géomécaniques au contact béton -fondation. La figure ci-après donne la coupe type du barrage au niveau du fond de vallée "figure1".

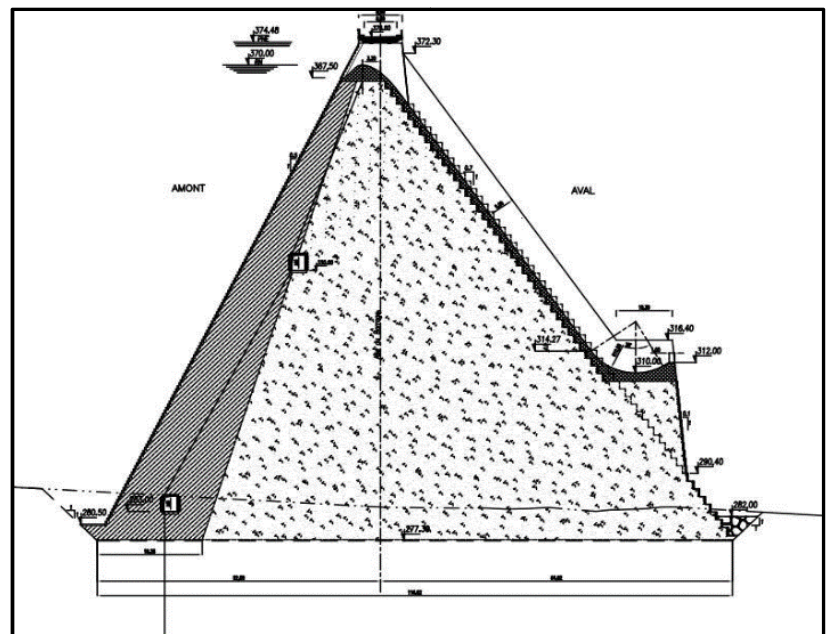

Fig.1. Coupe type au niveau de fond de vallée

La préparation des agrégats (concassage criblage) est faite sur le site. L'expérience montre que toutes les manipulations et les stockages ont tendance à générer des fines plastiques qui ne sont pas favorables à la résistance du béton.

Le BCR est mis en place selon la technique des couches inclinées rive-rive à $7 \%$. Les opérations sont continuées, depuis la préparation de la banquette de 20 cm d'épaisseur au contact de l'ancien BCR jusqu'à la 
mise en place de la couche elle-même. Chaque levée présente une épaisseur de 3 mètres. Cette hauteur correspond à la hauteur des coffrages qui déterminent les marches amont et aval. La technique de construction par couches inclinées élimine en grande partie le risque d'occurrence d'une couche horizontale faible, mince et continue ce qui est toujours à craindre accidentellement dans une région où les agrégats sont schisteux.

Le nettoyage de la surface de BCR est réalisé à la soufflette eau-air comprimé. Les agrégats sont juste dégagés en prévision du mortier de liaison. L'entrepreneur met en place environ $1500 \mathrm{~m} 3$ /jour à raison d'environ 8 heures par couche inclinée en partie inférieure.

\section{FORMULATION DU BETON}

Le béton est réalisé en exploitant au maximum les ressources du site. Le bassin versant est géologiquement homogène avec une dominante de schistes argileux. Il était illusoire de rechercher des gîtes à granulats qui seraient pauvres en fines argileuses. La préférence a été donnée à l'exploitation des zones à proximité immédiate du lit mineur de l'oued en évitant les pieds des versants. Il y a donc des matériaux schisteux dans les alluvions de la rivière. De plus, l'examen des matériaux sortant de la du malaxeur montre que ceux-ci présentent fréquemment une pellicule de fines argileuses qui n'est pas favorable à une bonne adhérence du liant. Leur élimination nécessite un lavage efficace et couteux si l'objectif est exagérément exigeant.

La formule retenue est la suivante :

Graves calcaire 0/16 $343 \mathrm{Kg}$ dont $16 \%$ de fines soit 55 $\mathrm{Kg}$ (carrière El Hajeb).

Graves de l'oued Bath 0/16 $937 \mathrm{Kg}$ dont $13 \%$ de fines soit $122 \mathrm{Kg}$.

$16 / 31.5$ de l'oued $478 \mathrm{Kg}$.

$31,5 / 63$ de l'oued $529 \mathrm{Kg}$.

Ciment CPJ $4590 \mathrm{Kg}$ (usine HOLCIM de fès).

Eau de l'oued Bath $134 \mathrm{Kg}$.

Adjuvant Plastiroute M 0,7\%.

L'objectif de résistance est fixé à $7 \mathrm{MPa}$ valeur minimum. La contrainte maximale de poids propre, qui est aussi sensiblement la contrainte de service est égale à 2,36 MPa, soit $33.6 \%$ de la résistance minimale exigée du BCR.

\section{LES ESSAIS DE LABORATOIRE SUR ECHANTILLON ET SUR CAROTTES.}

Les échantillons étaient prélevés régulièrement et soumis à des essais de compression simple et de traction (essai brésiliens).

Pour évaluer le module du BCR, une campagne de 9 échantillons a été programmée sur des BCR de plus de 90 jours. Tous les prélèvements ont été effectués le 13 Novembre 2014.Trois carottes de diamètre $175 \mathrm{~mm}$ extraite du massif de BCR entre la levée 19 et la levée 21, entre la cote 334,60 et la cote 341,50 NGM.Trois carottes de diamètre $137 \mathrm{~mm}$ extraite du massif de BCR entre la levée 19 et la levée 21 , entre la cote 334,44 et la cote 339,50 NGM.

Les échantillons proviennent des forages FCB16 et FCB18.Trois carottes de diamètre $153 \mathrm{~mm}$ carottés dans des éprouvettes de laboratoire (diamètre $25 \mathrm{~mm}$, hauteur $50 \mathrm{~mm}$ ) conservées sur le chantier du barrage et correspondant au BCR entre la levée 19 et la levée 20.

Des carottes de BCR ont été prélevées en parallèle pour chaque diamètre et chaque levée de la mise en place pour déterminer la résistance à la compression.Trois essais ont été réalisés sur des carottes de diamètre 137 prélevées le 13/11/2014, trois essais ont été réalisés sur des carottes de diamètre 175 prélevées le 12/11/2014 et trois essais ont été réalisés par carottage en diamètre 153 d'échantillons de dimension plus importante réalisés et conservés au laboratoire du chantier.

La photo ci-dessous montre une des carottes qui ont servi aux essais.

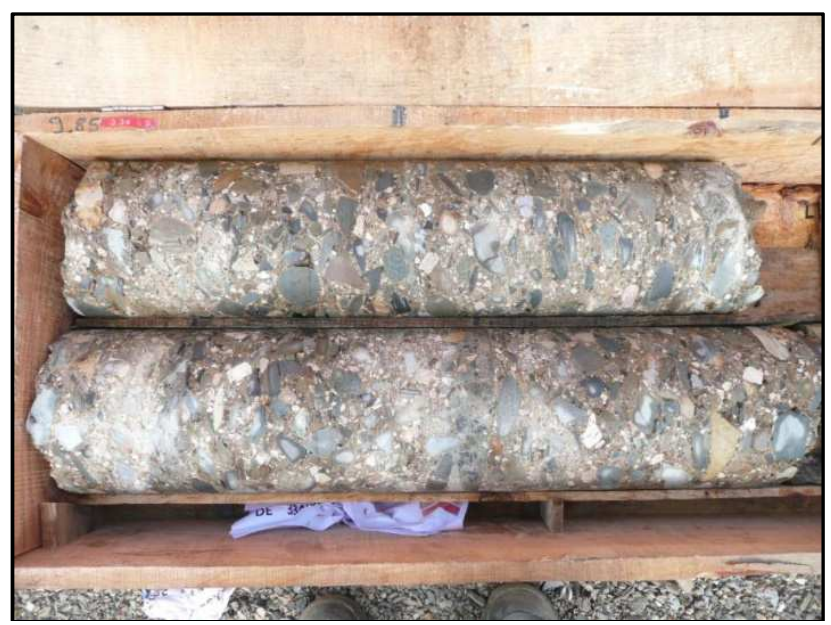

Les essais à 90 jours ont été réalisés en mars et juin 2014. Les essais réalisés le 1 décembre 2014 correspondent à des bétons coulés entre décembre 2013 et mars 2014. Ils ont donc un âge compris entre 8 mois et 12 mois.

Les essais suivent la norme ASTM C469-02. Des cycles de chargement et de déchargement en compression sont effectués sur des éprouvettes cylindriques d'élancement 2, par augmentation de la charge selon une vitesse constante de $0,05 \mathrm{MPa} / \mathrm{s}$ pour atteindre $60 \%$ de la charge maximale de rupture. Les déformations correspondantes sont déterminées par enregistrement des déplacements par des extensomètres électroniques.

Un essai de compression simple est d'abord réalisé pour déterminer la charge maximale à la rupture.Une adaptation du processus de mesure a été effectué pour tenir compte du fait que les éprouvettes testées montrent lors du premier cycle de chargement une déformation résiduelle qui varie de 50 à $400 \mu \mathrm{m} / \mathrm{m}$. Cette déformation résiduelle est apparue pour toutes les éprouvettes testées. Pourtant les cycles 2 et 3 montrent une réversibilité quasi parfaite avec des déformations résiduelles inférieures à $20 \mu \mathrm{m} / \mathrm{m}$. Les valeurs demodule donnée ci- dessous correspondent à $40 \%$ de la charge à la rupture pour le $3^{\circ}$ cycle. 
Un échantillon, le 24/1, donne un résultat de 10,5 GPa. Un échantillon, le 21/1 donne un résultat de 14,1 $\mathrm{GPa}$. Tous les autres essais donnent des résultats entre 17 et $21 \mathrm{GPa}$, avec une moyenne de $19 \mathrm{GPa}$.

Les essais sont effectués pour des déformations comprises entre 10-4 et 5. 10-4.

$\mathrm{Si}$ on adopte une résistance maximale moyenne de Rc $=9 \mathrm{MPa}$ et le module $\mathrm{E}=19 \mathrm{GPa}$ alors on peut en déduire que la rupture se produit pour une déformation limite de $\epsilon=9 / 19000=4,710-4$, ce qui est cohérent avec le champ de déformation utilisé pour les essais alternés permettant la détermination du module.

Le tableau suivant indique les résultats (la pente des courbes) pour les différents types d'éprouvettes, en distinguant les chargements successifs.

\begin{tabular}{|c|c|c|c|}
\hline éprouvette & $\begin{array}{c}\text { Premier } \\
\text { chargement }\end{array}$ & $\begin{array}{c}\text { Deuxième } \\
\text { chargement }\end{array}$ & $\begin{array}{c}\text { Troisième } \\
\text { chargement }\end{array}$ \\
\hline $20 / 1$ & 14,5 & 17,5 & 18,3 \\
\hline $20 / 3$ & 14,7 & 17,6 & 17,8 \\
\hline $21 / 1$ & 9,1 & 13,9 & 14,1 \\
\hline $21 / 2$ & 13 & 16,8 & 17,3 \\
\hline $21 / 5$ & 17,6 & 20,7 & 20,8 \\
\hline $24 / 1$ & 5,8 & 10 & 10,5 \\
\hline mov & 13,8 & 17,3 & 17,66 \\
\hline & & & 21 \\
\hline $436 / 13$ & 16,1 & 20,8 & 17,2 \\
\hline $440 / 14$ & 12,8 & 17,1 & 20,8 \\
\hline $454 / 14$ & 17,6 & 20,7 & 19,67 \\
\hline mov & 15,5 & 19,53 & \\
\hline
\end{tabular}

Dans tous les cas, les cycles alternés témoignent d'un comportement parfaitement élastique, les cycles 2 et 3 donnent des résultats très voisins. Le premier cycle témoigne d'un comportement irréversible qui peut correspondre soit à l'ajustement de l'échantillon dans la machine, soit à un comportement réel correspondant à un premier chargement. Entre le premier chargement et les chargements suivants, on constate une augmentation de $25 \%$.

Les carottes prélevées dans le corps du barrage étaient déjà chargées par le poids propre du barrage. Le BCR a été mis en place entre décembre 2013 et Mar 2014 entre la cote 334,6 et la cote 341,5 et le prélèvement par carottage a été fait le 13 novembre 2014 alors que le béton était en cours de mise en place à la cote 353,50. La charge de poids propre est donc comprise entre 12 mètres et 18 mètres. On constate que le module mesuré dans les échantillons carottés en place est inférieur de $11 \%$ à celui mesuré sur carottage d'échantillon en laboratoire.

Les mesures sur échantillons carottés in situ donnent des résultats un peu plus faibles et correspondent probablement mieux à la réalité physique du barrage que les mesures sur échantillon de laboratoire.

La différence entre le premier chargement et les suivants mérite réflexion. Dans la mesure où les chargements alternés sont relativement rapides, on pourrait considérer que le module mesuré est assez proche du module dynamique qui s'établirait ainsi vers 17 à $18 \mathrm{GPa}$.
Le module statique est très vraisemblablement supérieur au module de premier chargement, soit 13 à 14 GPa.

\section{ANALYSE DES ESSAIS CROSS-HOLE}

Trois doublets de forage ont été réalisés dans le corps du barrage. Leur implantation est schématisée sur la figure ci-dessous.

Trois mesures de vitesse des ondes de compression et de cisaillement ont été réalisées du 20 au 23 mai 2015 dans ces doublets par la méthode du cross-hole. Cette méthode consiste à émettre un train d'onde dans un forage et à mesurer le temps qu'il met pour atteindre l'autre forage en plaçant un géophone à la même cote. Les doublets de forages sont éloignés de 4,20 mètres pour les essais $\mathrm{CH} 1$ dans le plot 16 à la cote 293,5 et $\mathrm{CH} 2$ dans le plot 14 à la cote 296,5 , et 3,02 mètres pour le $\mathrm{CH} 3$, dans le plot 13 à la cote 429,5.

La masse volumique est supposée égale à $\rho=2400$ $\mathrm{Kg} / \mathrm{m} 3$.

Les essais cross-hole exigent des forages bien réalisés équipés de tube PVC parfaitement étanches (bouchon de pied), scellés très soigneusement au coulis de ciment.

La sonde d'émission est constituée de deux marteaux placés de part et d'autre du dispositif de placage, dans un corps de sonde. Le dispositif de placage est constitué d'un obturateur pneumatique disposé à l'intérieur d'un tube fendu (type sonde Ménard) assurant le placage et le couplage à la paroi du tubage. Une fois descendue à la cote d'essai dans le forage, la sonde est plaquée contre la paroi par l'obturateur. L'opérateur augmente la pression dans le circuit. Lorsqu'elle atteint une valeur d'environ 25 à 35 bars, le premier marteau est libéré et frappe l'enclume qui transmet l'onde sismique au terrain par l'obturateur. Le déclenchement du premier marteau arme le second marteau qui est libéré à son tour et émet une onde inverse. Grace à la propriété de polarisation de l'onde de cisaillement, cette frappe inverse provoque, sur la composante verticale, une onde $S$ inversée par rapport à celle émise par le premier marteau. Cela permet d'identifier l'arrivée de l'onde $\mathrm{S}$ avec plus de précision.

La sonde de réception est munie de capteurs tridirectionnels (Un capteur vertical et deux capteurs horizontaux). Le capteur vertical mesure principalement l'onde de cisaillement S. Les capteurs horizontaux mesurent principalement l'onde de compression P.

La déviation des forages est mesurée à l'aide d'une sonde inclinométrique Götzl ou équivalent afin de déterminer la distance entre le point d'émission et le point de réception pour chaque profondeur.

On appelle onde $\mathrm{P}$ l'onde de compression et onde $\mathrm{S}$ l'onde de cisaillement. Les vitesses de propagation sont respectivement $\mathrm{Vp}$ et Vs.

$\mathrm{Vp}$ et Vs sont reliées par le coefficient de poisson :

$v=(V p 2-2 V s 2) /(2(V p 2-V s 2))$

Les modules sont respectivement Module de compression

$E=2 . \rho . V s 2 .(1+v)$ 
Module de cisaillement $\mathrm{G}=\rho$. Vs2

Le fond de fouille est situé à la cote 277,30 . Les levées sont espacées de 3 mètres.L'identification des ondes $\mathrm{P}$ ne présente pas de difficulté alors que les ondes $\mathrm{S}$ sont plus difficilement identifiables car elles sont parasitées par les terrains encaissants qui provoquent une atténuation importante du signal, notamment pour le récepteur le plus éloigné de la source. Cela conduit à une incertitude sur la détermination du coefficient de Poisson. Comme la valeur de ce dernier est assez bien connu, et vraisemblablement assez constante dans le massif, on peut le prendre comme donnée et en déduire une autre valeur possible pour la vitesse Vs.

Les résultats sont :

\section{Cross- hole CH1 :}

Dans le BCR, les vitesses Vp varient entre $2917 \mathrm{~m} / \mathrm{s}$ et $3200 \mathrm{~m} / \mathrm{s}$, soit une valeur moyenne de $3056 \mathrm{~m} / \mathrm{s}$. les vitesses VS varient entre $1400 \mathrm{~m} / \mathrm{s}$ et $1780 \mathrm{~m} / \mathrm{s}$ soit une valeur moyenne de $1580 \mathrm{~m} / \mathrm{s}$.

Dans le rocher proche du contact béton rocher, donc injecté par les injections de consolidation, $\mathrm{Vp}$ varie entre $3500 \mathrm{~m} / \mathrm{s}$ et $4038 \mathrm{~m} / \mathrm{s}$ soit une valeur moyenne de 3829 $\mathrm{m} / \mathrm{s}$, Vs varie entre $1500 \mathrm{~m} / \mathrm{s}$ et $1850 \mathrm{~m} / \mathrm{s}$ soit une valeur moyenne de $1700 \mathrm{~m} / \mathrm{s}$.

\section{Cross-hole $\mathrm{CH} 2$ :}

Dans le BCR, les vitesses Vp varient entre $2950 \mathrm{~m} / \mathrm{s}$ et $3141 \mathrm{~m} / \mathrm{s}$, soit une valeur moyenne de $3077 \mathrm{~m} / \mathrm{s}$. les vitesses VS varient entre $1450 \mathrm{~m} / \mathrm{s}$ et $1820 \mathrm{~m} / \mathrm{s}$ soit une valeur moyenne de $1635 \mathrm{~m} / \mathrm{s}$.

Dans le rocher proche du contact béton rocher, donc injecté par les injections de consolidation, $\mathrm{Vp}$ varie entre $3500 \mathrm{~m} / \mathrm{s}$ et $4038 \mathrm{~m} / \mathrm{s}$ soit une valeur moyenne de 3829 $\mathrm{m} / \mathrm{s}$, Vs varie entre $1850 \mathrm{~m} / \mathrm{s}$ et $1900 \mathrm{~m} / \mathrm{s}$ soit une valeur moyenne de $1875 \mathrm{~m} / \mathrm{s}$.

\section{Cross-hole $\mathrm{CH} 3$ :}

Dans le BCR, les vitesses Vp varient entre $2860 \mathrm{~m} / \mathrm{s}$ et $3300 \mathrm{~m} / \mathrm{s}$, soit une valeur moyenne de $3041 \mathrm{~m} / \mathrm{s}$. les vitesses VS varient entre $1500 \mathrm{~m} / \mathrm{s}$ et $1780 \mathrm{~m} / \mathrm{s}$ soit une valeur moyenne de $1660 \mathrm{~m} / \mathrm{s}$. Quelques valeurs de Vs sont faibles $(1540 \mathrm{~m} / \mathrm{s})$.

Le tableau suivant indique les différentes valeurs et calcul Vs en faisant l'hypothèse que $v$ est égal à 0,2 , ce qui ne change pas significativement Vs et reste dans le champ de l'incertitude de la mesure.

\begin{tabular}{|c|c|c|c|c|c|c|c|c|}
\hline & VP & & VS/VP & VS/VP carré n & & ro & E & Vs pour nu 0,2 \\
\hline CH1 min & 2917 & 1400 & 0,47994515 & 0,23034735 & 0,3503563 & 2400 & $1,2704 E+10$ & 1326,80 \\
\hline CH1 max & 3200 & 1780 & 0,55625 & 0,30941406 & 0,27597715 & 2400 & $1,9405 E+10$ & 1782,23 \\
\hline CH1 moy & 3056 & 1580 & 0,51701571 & 10,26730524 & 0,31758758 & 2400 & $1,5788 E+10$ & 1539,68 \\
\hline $\mathrm{CH} 2 \mathrm{~min}$ & 2950 & 1450 & 0,49152542 & 2) 0,24159724 & 0,3407197 & 2400 & $1,3531 \mathrm{E}+10$ & 1391,60 \\
\hline CH $2 \max$ & 3141 & 1820 & 0,5794333 & 3. 0,33574295 & 0,24727935 & 2400 & $1,9831 \mathrm{E}+10$ & 1933,88 \\
\hline CH2 moy & 3077 & 1635 & 0,53136172 & 2. 0,28234527 & 0,30328613 & 2400 & $1,6723 E+10$ & 1626,31 \\
\hline $\mathrm{CH} 3 \mathrm{~min}$ & 2860 & 1500 & 0,52447552 & 0,27507458 & 0,31027388 & 2400 & $1,4151 \mathrm{E}+10$ & 1584,43 \\
\hline CH3 max & 3300 & 1780 & 0,53939394 & 4,29094582 & 0,29483527 & 2400 & $1,9692 \mathrm{E}+10$ & 1675,85 \\
\hline CH3 moy & 3041 & 1660 & 0,54587307 & 0,29797741 & 0,28777221 & 2400 & $1,7033 \mathrm{E}+10$ & 1716,35 \\
\hline rocher CH1max & 4038 & 1850 & 0,4581476 & 0,20989922 & 0,36716934 & 2400 & $2,246 \mathrm{E}+10$ & 1209,02 \\
\hline rocher CH1min & 3500 & 1500 & 0,42857143 & 0,18367347 & 0,3875 & 2400 & $1,4985 \mathrm{E}+10$ & 1057,96 \\
\hline rocher CH2 max & 4038 & 1900 & 0,47052997 & 0,22139845 & 0,35782301 & 2400 & $2,3528 E+10$ & 1275,26 \\
\hline rocher CH2 min & 3500 & 1850 & 0,52857143 & $\begin{array}{ll}3 & 0,27938776\end{array}$ & 0,30614557 & 2400 & $2,1457 E+10$ & 1609,27 \\
\hline
\end{tabular}

Il est à noter que la vitesse $\mathrm{Vp}$ dans le rocher schisteux est relativement élevée, entre $3500 \mathrm{~m} / \mathrm{s}$ et 4000 $\mathrm{m} / \mathrm{s}$. Ce schiste est relativement peu compressible. C'est son angle de frottement assez faible qui a conduit à dessiner un projet quasi symétrique qui sollicite peu la fondation en cisaillement. Nous avons déjà constaté dans des formations géologiques analogues des déplacements très faibles à la mise en eau du barrage, donc des modules du rocher de fondation plutôt élevés. Par ailleurs, les injections de consolidation ont été réalisés avec beaucoup de soin et à relativement haute pression d'injection, et donc, semble-t-il avec efficacité. Les injections améliorent notablement le module de déformation des rochers fissurés.

Les figures suivantes montrent que le module croit avec la profondeur pour les Crosshole $\mathrm{CH} 1$ et $\mathrm{CH} 2$.

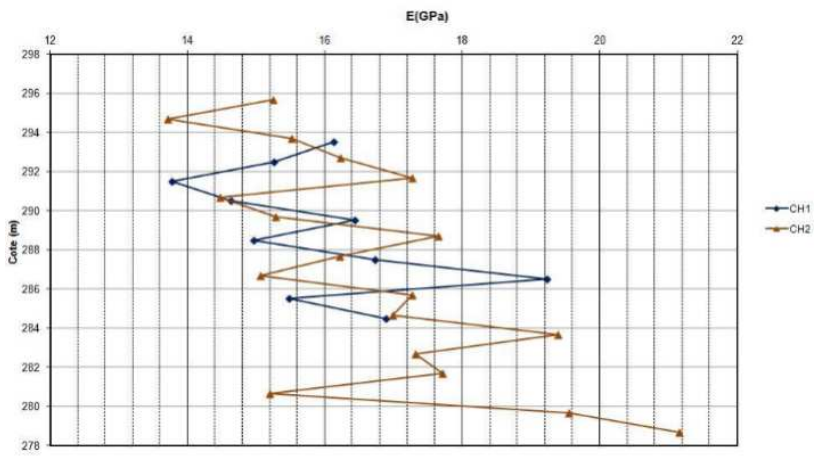

Cette tendance est sensiblement linéaire. $\mathrm{Si}$ on suppose que cette évolution est due à l'âge du béton, alors la progression du BCR est d'environ une levée de 3 mètres par mois et passe de 14,5 GPa à $18 \mathrm{GPa}$ entre la cote 280 et la cote 295 , soit en 5 mois. La valeur du module serait donc de la forme :

$E=14,5+n j / 150 \times 3,5$

nj étant le nombre de jours

14,5 est le module à la cote 296 , c'est-à-dire le 19 juin 2012, soit pour un béton dont l'âge est de presque 3 ans, précisément 1068 jours, et $18 \mathrm{GPa}$ serait le module pour un béton plus vieux d'au moins 5 mois.

Une telle variation du module pour un béton déjà âgé est peu vraisemblable. Le tableau et la courbe suivante donne l'évolution de la résistance à la compression des carottes extraites des éprouvettes avec l'âge qui donne une idée de l'évolution du module avec l'âge :

A 7 jours $\sigma=6,3 \mathrm{MPa}$

A 28 jours $\sigma=8,1 \mathrm{MPa}$

A 90 jours $\sigma=9,4 \mathrm{MPa}$

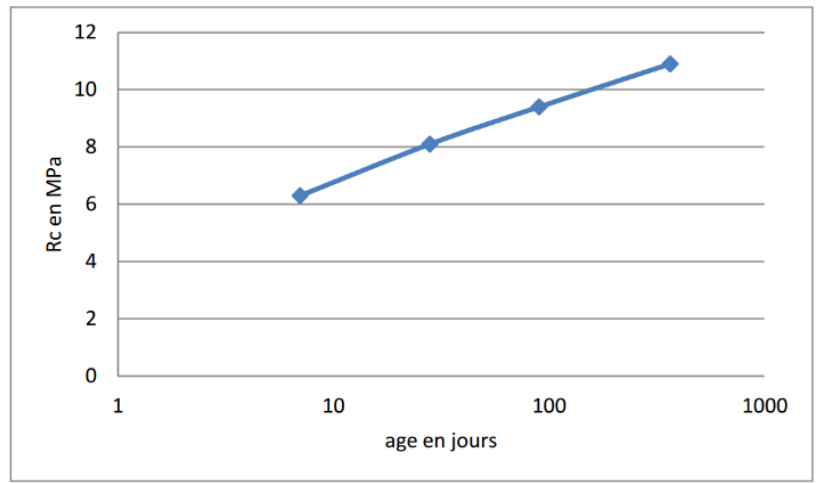

Au-delà de 1000 jours, la variation est très lente.

Cette progression du module est probablement due à la proximité croissante du rocher dont la vitesse est plus élevée que dans le béton.

On peut admettre en fonction des résultats des mesures, que le module du rocher est voisin de $20 \mathrm{GPa}$ et 
que le module du BCR est supérieur à $14 \mathrm{GPa}$ et compris entre 15 et $16 \mathrm{GPa}$. Ce module dynamique prend en compte le principe de mise en place du BCR, par couches de $0,30 \mathrm{~m}$ d'épaisseur inclinées à $7 \%$, sans traitement du joint, ce qui peut conduire à une diminution du module pour l'ensemble de la masse du béton.

\section{MESURES DE VITESSES SUR EPROUVETTES}

En novembre 2013, des essais de mesures de vitesses d'onde avaient été réalisés sur le chantier, sur des éprouvettes de BCR.Différents essais ont été réalisés :

a) Vitesses de propagation d'onde mesurées sur carottes extraites du BCR in situ :

\begin{tabular}{|l|l|}
\hline BCR in situ & Vitesse en $\mathrm{m} / \mathrm{s}$ \\
\hline Nombre & 5 \\
\hline Max & 3540 \\
\hline Moyenne & 3000 \\
\hline Min & 2300 \\
\hline
\end{tabular}

b) Vitesses de propagation d'onde mesurées sur des carottes extraites sur des blocs réalisés au laboratoire.

\begin{tabular}{|l|l|}
\hline BCR laboratoire & Vitesse en $\mathrm{m} / \mathrm{s}$ \\
\hline Nombre & 5 \\
\hline Max & 4090 \\
\hline Moyenne & 3960 \\
\hline Min & 3820 \\
\hline
\end{tabular}

Les mesures faites sur les carottes du 21/03/2013, levée 14/couche 1 , ont une résistance à la compression Rc $=10,2 \mathrm{MPa}$ à 90 jours. Le test a été réalisé le 02/09/2013 soit après 160 jours. La moyenne est égale à $3320 \mathrm{~m} / \mathrm{s}$ avec une dispersion assez faible.

Les mesures faites sur les carottes du10/04/2013, levée $14 /$ couche 1 , ont une résistance à la compression Rc = 9,8 $\mathrm{MPa}$ à 90 jours. Le test a été réalisé le 02/09/2013 soit après 140 jours. La moyenne est égale à $2515 \mathrm{~m} / \mathrm{s}$. Cette valeur basse est un peu surprenante d'autant que la résistance à la compression y est nettement supérieure à la moyenne. Dans le cas des mesures sur carottes extraites du corps du barrage, les valeurs mesurées, donnent des valeurs proches des valeurs mesurées par les essais cross-hole.

\section{MODULE STATIQUE ET MODULE DYNAMIQUE}

En principe, le module mesuré en laboratoire par un essai de compression simple est le module statique et le module mesuré au cross-hole est le module dynamique. Il est très généralement admis que le module dynamique est plus élevé que le module statique. Des anciens travaux montrent que sur des bétons beaucoup plus dosés que notre BCR. Il donne cependant des informations très utiles. Les éprouvettes saturées en eau libre (ce qui est le cas du BCR des barrages) présentent une augmentation de résistance à la traction importante en fonction de la vitesse de chargement. En revanche, le module d'Young varie très peu avec la vitesse de chargement, pas plus de $10 \%$ à $15 \%$ pour une multiplication de la vitesse de chargement de 5 ordres de grandeurs (page 75).

Dans le cas du BCR, la structure d'hydrate est lâche. Le squelette n'a été constitué qu'après la formation des cristaux d'hydrates entre les grains assez lointains

Le module mesuré par la méthode du cross-hole fournit une valeur du module dynamique pour la masse du béton prenant en compte le système de construction de cette masse qui n'est pas parfaitement homogène, car elle est formée de couches inclinées, de joints entre couches non traités au mortier et de joints entre levées de 3 mètres traités au mortier.

Les essais alternés en laboratoire montrent des valeurs un peu plus élevées pour le béton conservé en laboratoire que pour le béton carotté en place et qui intègre les spécificités de la mise en place (construction par couches inclinées et joints non traités).

Les essais alternés sont relativement rapides et dans une gamme de déformation plutôt supérieur aux déformations dynamiques lors du séisme de dimensionnement.

On pourrait donc retenir pour la masse du barrage, un module statique de 13 à $14 \mathrm{GPa}$ et un module dynamique de 15 à 16 Gpa, mais il n'est pas impossible que les deux soient très voisins.

Il n'y a pas d'argument pour adopter un module statique plus bas que $13 \mathrm{GPa}$ et il n'y a pas d'arguments pour adopter un module dynamique supérieur à $16 \mathrm{GPa}$.

Pour caractériser le module dynamique sous très faible déformation, on peut mesurer les vibrations naturelles du barrage, éventuellement sollicités, et en déduire les fréquences des premiers modes ce qui donne les modules du béton. Une telle mesure faite dans un pays voisin pour un barrage de profil et de hauteur voisins et une fondation assez similaire, indique un module d'environ 17 à $18 \mathrm{GPa}$.

\section{REFERENCES BIBLIOGRAPHIQUES}

1. J.M Medes et al. La technique du béton compacté au rouleau (BCR)-Possibilité d'application pour les grands barrages en Afrique -Sud ScienceTechnologie-56-62 (Janvier 1998).

2. Géologie et barrages en béton compacté au rouleau (BCR)- Bulletin of Engineering Geology and the Environment-The official journal of the IAEGVolume 55, Issue 1, pp 77-81 (April 1997)

3. André GOUBET-Béton compacté au rouleauREVUEFRANÇAISE DE GÉOTECHNIQUE-P4753- (1998).

4. C. Bobard, J.L.Mattiuzzo- Essais cross hole, nouveau développement pour la réalisation et l'interprétationjournées Nationales de Géotechnique et de Géologie de l'Ingénieur -Nancy (2016).

5. Standard Test Methods for Crosshole Seismic Testing- ASTM D 4428/D 4428M (Janvier 2014).

6. G Benrnard, Techniques géophysiques de haute résolution, Tomographie géophysique en forage, cours en ligne, Ecole Polytechnique de Montréal, 47p (2005). 